\title{
THE SET OF PRIMES DIVIDING THE LUCAS NUMBERS HAS DENSITY 2/3
}

\author{
J. C. Lagarias \\ Bell Laboratories \\ Murray Hill, NJ 07974
}

\section{Introduction}

There has been a good deal of study of the structure of the set of prime divisors of the terms $\left\{U_{n}\right\}$ of second order linear recurrences. M. Ward [14] showed that there are always an infinite number of distinct primes dividing the terms $\left\{U_{n}\right\}$, provided we exclude certain degenerate cases such as $U_{n}=2^{n}$. In fact, under the same circumstances it is believed that the set of primes dividing the terms $U=\left\{U_{n}\right\}$ of any nondegenerate second order linear recurrence has a positive density $d(U)$ depending on the recurrence. This can be proved under the asSption that the generalized Riemann hypothesis is true by a method analogous to Hooley's conditional proof of Artin's Conjecture for primitive roots. P. J. Stephens [13] has done this for a large class of such second-order recurrences.

The point of this paper is that there are special second order linear recurrences where it is possible to give an unconditional proof of the existence of a density. This was shown by Hasse [4] for certain special second order recurrences having a reducible characteristic polynomial, in the process of solving a problem of Sierpinski [12]. Sierpinski's problem concerns the existence of a density for the set of primes $p$ for which $\operatorname{ord}_{p} 2$ is even. This set of primes is exactly the set of primes dividing some term of the sequence $V_{n}=2^{n}+1$; this sequence satisfies the reducible second order linear recurrence $V_{n}=3 V_{n-1}-2 V_{n-2}$ with $V_{0}=2$ and $V_{1}=3$.

Theorem A. (Hasse) The set of primes $S_{V}=\left\{p: p\right.$ is prime and $p$ divides $2^{n}+1$ for some $\left.n \geq 0\right\}$ has density 2/3.

Hasse's result [4] actually covers all the sequences $\left\{a^{n}+1: n \geq 0\right\}$, where $a$ is an integer.

Here we observe that Hasse's method with some extra complications extends to cover certain secondorder linear recurrences with irreducible characteristic polynomial. The most interesting example of this phenomonon is the Lucas numbers $L_{n}$ defined by $L_{1}=2, L_{2}=1$ and the recurrence $L_{n+1}=L_{n}+L_{n-1}$. 
Theorem B. The set of primes $S_{L}=\left\{p: p\right.$ is prime and $p$ divides some Lucas number $\left.L_{n}\right\}$ has density $2 / 3$.

Theorem B can be alternatively derived from polynomial-splitting criteria of M. Ward [16] for membership in $S_{L}$; this is essentially the same proof.

Hasse's method applies to any irreducible second-order recurrence $\left\{U_{n}\right\}$ whose general term can be written

$$
U_{n}=\alpha \theta^{n}+\bar{\alpha} \bar{\theta}^{n}
$$

where $\alpha$ and $\theta$ are in the quadratic field $K$ generated by the roots of the characteristic polynomial of $\left\{U_{n}\right\}$, and $\bar{\alpha}, \bar{\theta}$ are the algebraic conjugates of $\alpha, \theta$ in $K$, provided that:

(i) $\frac{\theta}{\bar{\theta}}= \pm \phi^{k}$ where $k=1$ or 2 for some $\phi$ in $K$.

(ii) $\quad \frac{\bar{\alpha}}{\alpha}=\zeta \phi^{j}$ where $\zeta$ is a root of unity in $K$ and $j$ is an integer.

The actual densities of the sets of primes obtained depend in an idiosyncratic way on $\alpha$ and $\theta$, which makes it awkward to state a general result. Some of the possible extra complications encountered are illustrated in the proof of the following result, concerning a particular recurrence discussed in Laxton [8].

Theorem C. Let $W_{n}$ denote the recurrence defined by $W_{0}=1, W_{1}=2$ and $W_{n}=5 W_{n-1}-7 W_{n-2}$. Then the set $S_{W}=\left\{p: p\right.$ divides $W_{n}$ for some $\left.n\right\}$ has density $5 / 8$.

The parameterized families of recurrences $A_{n}(m)$ and $B_{n}(m)$, both of which satisfy the recurrence

$$
U_{n}=m U_{n-1}-U_{n-2}
$$

with initial conditions $A_{0}(m)=B_{0}(m)=1$ and $A_{1}(m)=m+1, B_{1}(m)=m-1$, are also recurrences to which Hasse's method applies. In the case that $\varepsilon=\frac{1}{2}\left(m+\sqrt{m^{2}-4}\right)$ is the fundamental unit in $K=Q\left(\sqrt{\left.m^{2}-4\right)} \quad\right.$ the $\quad$ sets $\quad S_{A}(m)=\left\{p: p\right.$ divides $A_{n}(m)$ for some $\left.n\right\} \quad$ and $S_{B}(m)=\left\{p: p\right.$ divides $B_{n}(m)$ for some $\left.n\right\}$ each have density $1 / 3$. I omit the details.

I give a proof of Theorem A in Section 2 for comparison with the more involved details of the proofs of 
Theorem B and C in Sections 3 and 4, respectively.

\section{Proof of Theorem A}

The condition that $p \mid 2^{n}+1$ for some $n$ can be rewritten as:

$$
2^{n} \equiv-1(\bmod p) \text { is solvable }
$$

Now let $m=\operatorname{ord}_{p}$ 2, the least positive integer with

$$
2^{m} \equiv 1(\bmod p)
$$

Now (2.1) is solvable if and only if $m$ is even and the smallest solution to (2.1) in that case is $n=\frac{1}{2} m$. Now suppose $2^{j}$ exactly divides $p-1$. Then we have:

$$
2^{j} \| p-1 \text { and } \operatorname{ord}_{p} 2 \text { is odd } \Leftrightarrow 2^{\frac{p-1}{2^{j}}} \equiv 1(\bmod p)
$$

Hasse observes that the condition on the right side of (2.3) is a splitting condition for primes in a certain algebraic number field $K_{j}$; such sets of primes have a density by the Frobernius density theorem.

Consequently we proceed by decomposing the set $S_{V}$ into disjoint sets

$$
S_{V}=\bigcup_{j=1}^{\infty} S_{V}^{(j)}
$$

given by

$$
S_{V}^{(j)}=\left\{p: p \equiv 1+2^{j}\left(\bmod 2^{j+1}\right) \text { and } p \in S_{A}\right\} \text {. }
$$

We also define

$$
\bar{S}_{V}^{(j)}=\left\{p: p \equiv 1+2^{j}\left(\bmod 2^{j+1}\right) \text { and } p \notin S_{A}\right\}
$$

and observe $p \varepsilon \bar{S}_{V}^{(j)}$ if and only if $p \equiv 1+2^{j}\left(\bmod 2^{j+1}\right)$ and $(2.3)$ holds. To state Hasse's observation precisely, let $C_{j}$ denote the cyclotomic field $Q\left({ }^{2^{j}} \sqrt{ } 1\right)$, let $K_{j}=Q\left({ }^{j^{j}} \sqrt{ } \overline{1}^{-}{ }^{2^{j}} \sqrt{2}\right)$ and let $L_{j}=Q\left(2^{j+1} \sqrt{1}-2^{j} \sqrt{ } 2 \overline{)}\right.$.

Lemma 2.1. (1) The primes $p$ in $\bar{S}_{V}^{(j)}$ are exactly the primes $p$ that split completely in $L_{j}$ but not in $K_{j}$. 
(2) The primes $p$ in $\bar{S}_{V}^{(j)}$ have density $2^{-2 j}$ and those in $S_{V}^{(j)}$ have density $2^{-j}-2^{-2 j}$, i.e.

$$
\begin{aligned}
& \#\left\{p \leq x: p \varepsilon \bar{S}_{V}^{(j)}\right\} \sim 2^{-2 j} \frac{x}{\ln x}, \\
& \#\left\{p \leq x: p \varepsilon S_{V}^{(j)}\right\} \sim\left(2^{-j}-2^{-2 j}\right) \frac{x}{\ln x},
\end{aligned}
$$

as $x \rightarrow \infty$.

Proof. The fields $C_{j}=Q\left(2^{2^{j-1}} \sqrt{ }-\overline{1}\right), K_{j}=C_{j}\left(2^{j} \sqrt{ } 2\right)$ and $L_{j}=C_{j+1}\left(2^{j} \sqrt{2} \overline{)}\right.$ are all normal extensions of the rationals. The condition that the ideal ( $p$ ) split completely over a cyclotomic field $Q\left({ }^{m} \sqrt{1} \overline{)}\right.$ is well known to be $p \equiv 1(\bmod m)$ ([2], Lemma 4), hence $\left.p \equiv 1\left(\bmod 2^{j}\right)\right)$ holds if and only if $p$ splits completely in $C_{j}$. The condition that a prime ideal $p$ in $C_{j}$ split completely in the Kummer extension $K_{j}=C_{j}\left(2^{j} \sqrt{2}\right)$ is exactly that

$$
x^{2^{j}} \equiv 2(\bmod (p)) \text { for } x \in O_{j}
$$

be solvable over the ring of integers $O_{j}$ for $C_{j}$ ([2], Lemma 5). If $p$ is of degree 1 then any algebraic integer $x$ in $C_{j}$ is congruent to a rational integer $(\bmod p)$ so in this case equation (2.5) is solvable if and only if

$$
x^{2^{j}} \equiv 2(\bmod p) \text { for } x \in Z
$$

is solvable. By Euler's criterion (2.6) is solvable if and only if

$$
2^{\frac{p-1}{2^{\prime}}} \equiv 1(\bmod p)
$$

is solvable. This is exactly (2.3), and we have shown $(p)$ splits completely in $K_{j}$ iff $p \equiv 1\left(\bmod 2^{j}\right)$ and (2.7) holds. Similarly $(p)$ splits completely in $L_{j}$ iff $p \equiv 1\left(\bmod 2^{j+1}\right)$ and (2.7) holds. This proves (1).

To prove (2) we observe that for a normal extension $K / Q$ of degree $[K: Q]$ the set of primes $p$ that split completely in $K$ has density [ $K: Q]^{-1}$, which is a consequence of the prime ideal theorem (e.g. [6], p. 315 Theorem 4), a special case of both the Frobenius and Chebotarev density theorem. Now $\left[C_{j}: Q\right]=2^{j-1}$, $\left[K_{j}: Q\right]=2^{2 j-1}$ and $\left[L_{j}: Q\right]=2^{2 j}$. The set of primes in $\bar{S}_{V}^{(j)}$ is the difference of a set of primes of density $2^{-(2 j-1)}$ less a class of primes contained in it of density $2^{-2 j}$, hence has density $2^{-2 j}$. Finally the primes in $S_{V}^{(j)}$ are the difference of the class of primes $\left\{p \equiv 1+2^{j}\left(\bmod 2^{j+1}\right)\right\}$ of density $2^{-j}=\left[C_{j}: Q\right]^{-1}-\left[C_{j+1}: Q\right]^{-1}$, and the class of primes $\bar{S}_{V}^{(j)}$ of density $2^{-2 j}$ contained in it. This proves 
(2).

To complete the proof of Theorem A, we observe that for any fixed $m$,

$$
\bigcup_{j=1}^{m} S_{V}^{(j)} \subseteq S_{V} \subseteq \mathbf{P}-\bigcup_{j=1}^{m} \bar{S}_{V}^{(j)}
$$

where $\mathbf{P}$ denotes the set of all primes. Using (2) of Lemma 2.1, the first inclusion gives

$$
\#\left\{p \leq x: p \varepsilon S_{V}\right\} \geq\left(\frac{2}{3}-2^{-m}-\frac{4}{3} 2^{-2 m}\right) \frac{x}{\ln x}+O\left(\frac{x}{\ln x}\right)
$$

as $x \rightarrow \infty$, since all the $S_{V}^{(j)}$ are disjoint. The second inclusion gives

$$
\#\left\{p \leq x: p \varepsilon S_{V}\right\} \leq\left(\frac{2}{3}+\frac{4}{3} 2^{-2 m}\right) \frac{x}{\ln x}+O\left(\frac{x}{\ln x}\right) .
$$

as $x \rightarrow \infty$. Letting $m \rightarrow \infty$ shows that

$$
\#\left\{p \leq x: p \varepsilon S_{V}\right\} \sim \frac{2}{3} \frac{x}{\ln x}
$$

Remarks. (1) By a careful analysis of error terms in this argument using an effective version of the Chebotanev density theorem, Odoni [11] has proved the stronger result that:

$$
\#\left\{p \leq x: p \varepsilon S_{V}\right\}=\frac{2}{3} \operatorname{Li}(x)+O\left(\operatorname{Li}(x) \exp \left(-c \frac{\ln \ln x}{\ln \ln \ln x}\right)\right)
$$

where $\operatorname{Li}(x)=\int_{2}^{x} \frac{d t}{\ln t}$.

(2) The sets $S_{V}^{(j)}$ are sets of primes determined by systems of polynomial congruences in the sense of [5, Theorems 1.1 and 1.2].

\section{Proof of Theorem B}

The Lucas numbers $L_{n}$ satisfy

$$
L_{n}=\varepsilon^{n}+\bar{\varepsilon}^{n}
$$

where $\varepsilon=\frac{1+\sqrt{5}-}{2}$ and $\bar{\varepsilon}=\frac{1-\sqrt{5}-}{2}$. Hence 


$$
\begin{aligned}
p \mid L_{n} & \Leftrightarrow \varepsilon^{n}+\varepsilon^{-1} \equiv \theta(\bmod (p)) \\
& \Leftrightarrow \theta^{n} \equiv-1(\bmod (p))
\end{aligned}
$$

where

$$
\theta=\frac{\varepsilon}{\bar{\varepsilon}}=-\varepsilon^{2}=-\frac{3+\sqrt{5}-}{2}
$$

and the congruences are in the ring $Z\left[\frac{1+\sqrt{5}-}{2}\right]$ of algebraic integers in $Q\left(\sqrt{5} \overline{)}\right.$. Thus $S_{L}$ is exactly the set of primes $p$ for which the exponential congruence over $Z\left[\frac{1+\sqrt{5}-}{2}\right]$

$$
\theta^{x} \equiv-1(\bmod (p))
$$

is solvable for some integer $x$.

We now proceed analogously to the proof of Theorem A. We must treat several cases according to the behavior of the ideal $(p)$ in $Z\left[\frac{1+\sqrt{5}-}{2}\right]$. If $p \equiv \pm 1(\bmod 5)$ then $(p)=\pi \bar{\pi}$ splits into two conjugate degree 1 prime ideals, while if $p \equiv \pm 2(\bmod 5)$ then $(p)$ is a degree 2 prime ideal in $Z\left[\frac{1+\sqrt{5}-}{2}\right]$. Let $S_{L}=S_{A} \cup S_{B}$ where

$$
S_{A}=\left\{p: p \varepsilon S_{L} \text { and } p \equiv \pm 1(\bmod 5)\right\}
$$

and

$$
S_{B}=\left\{p: p \varepsilon S_{L} \text { and } p \equiv \pm 2(\bmod 5)\right\} .
$$

Case 1. The primes in $S_{A}$ have density $\frac{5}{12}$.

Write $(p)=\pi \bar{\pi}$ in $Z\left[\frac{1+\sqrt{5}-}{2}\right]$. In this case (3.3) is equivalent to

$$
\theta^{x} \equiv-1\left(\bmod \pi_{1}\right)
$$

being solvable. To see this, suppose (3.4) holds and apply the automorphism taking $\sqrt{5}$ to $-\sqrt{5}$ to (3.4) to get 


$$
\bar{\theta}^{x} \equiv-1\left(\bmod \bar{\pi}_{1}\right) .
$$

Since $\theta \bar{\theta}=1$ we have $\theta^{x} \bar{\theta}^{x}=1$ so (3.5) implies

$$
\theta^{x} \equiv-1\left(\bmod \bar{\pi}_{1}\right)
$$

Combining this with (3.4) shows (3.3) holds. The reverse direction is clear.

Now we have the equivalence

$$
\operatorname{ord}_{\pi_{1}} \theta \text { is even } \Leftrightarrow \theta^{x} \equiv-1(\bmod (p)) \text { is solvable . }
$$

If $p \equiv 1+2^{j}\left(\bmod 2^{j+1}\right)$ we obtain

$$
2^{j} \| p-1 \text { and } \operatorname{ord}_{\pi} \theta \text { is odd } \Leftrightarrow \theta^{\frac{p-1}{2^{\prime}}} \equiv 1\left(\bmod \pi_{1}\right)
$$

This leads us to split $S_{A}$ into the disjoint union of sets

$$
S_{A}=\bigcup_{j=1}^{\infty} S_{A}^{(j)}
$$

where

$$
S_{A}^{(j)}=\left\{p: p \equiv 1+2^{j}\left(\bmod 2^{j+1}\right) \text { and } \operatorname{ord}_{\pi_{1}} \theta \text { is even }\right\} .
$$

We set

$$
\bar{S}_{A}^{(j)}=\left\{p: p \equiv 1+2^{j}\left(\bmod 2^{j+1}\right) \text { and } \operatorname{ord}_{\pi_{1}} \theta \text { is odd }\right\} .
$$

The associated fields are $K_{j}^{*}=Q\left(2^{j} \sqrt{1}, \sqrt{5} \overline{, 2}^{j} \sqrt{ } \bar{\theta}\right)$ and $L_{j}^{*}=Q\left(2^{2^{j+1}} \sqrt{1} \overline{1} \sqrt{5} \overline{,^{2^{j}}} \sqrt{\theta} \overline{)}\right.$.

Lemma 3.1. (1) $\bar{S}_{A}^{(1)}$ is empty. For $j \geq 2$ the primes $p$ in $\bar{S}_{A}^{(j)}$ are exactly the primes that split completely in $K_{j}^{*}$ and which do not split completely in $L_{j}^{*}$.

(2) The primes in $\bar{S}_{A}^{(1)}$ and $S_{A}^{(1)}$ have densities 0 and $1 / 4$, respectively. For $j \geq 2$ the primes in $\bar{S}_{A}^{(j)}$ have density $2^{-2 j}$ and those in $S_{A}^{(j)}$ have density $2^{-j-1}-2^{-2 j}$.

Proof. Similar to that of Lemma 2.1. The relation $\theta=-\varepsilon^{2}$ leads to $K_{1}^{*}=L_{1}^{*}=Q(\sqrt{-1}, \sqrt{5} \overline{)}$; this causes $S_{A}^{(1)}$ to be empty. For $j \geq 2$ one checks that $\left[K_{j}^{*}: Q\right]=2^{2 j-1}$ and $\left[L_{j}^{*}: Q\right]=2^{2 j}$. In fact for $j \geq 2, \quad K_{j}^{*}=Q\left(\omega_{j}, \sqrt{5}, \phi_{j-2} \sqrt{\left(\overline{\omega_{j} \phi_{j-2}}\right)} \quad\right.$ where $\quad \omega_{j}=2^{j-1} \sqrt{-1} \quad$ and $\quad \phi_{j-2}=2^{2^{-2}} \sqrt{\varepsilon}, \quad$ and 
$L_{j}^{*}=Q\left(\omega_{j+1}, \sqrt{5}, \phi_{j-1}\right)$. Finally note that the set $S_{A}^{(j)} \cup \bar{S}_{A}^{(j)}=\{p: p \equiv \pm 1(\bmod 5)\}$ and $p \equiv 1+2^{j}\left(\bmod 2^{j+1}\right)$ has density $2^{-j-1}$.

As in the proof of Theorem A we find the primes in $S_{A}$ have density $\frac{1}{4}+\int_{j=2}^{\infty}\left(2^{-j+1}-2^{-2 j}\right)$ $=\frac{1}{2}-\frac{1}{12}=\frac{5}{12}$.

Case 2. The primes in $S_{B}$ have density $\frac{1}{4}$.

The primes $p \equiv \pm 2(\bmod 5)$ remain inert in $Z\left[\frac{1+\sqrt{5}-}{2}\right]$, and in this case

$$
\theta^{x} \equiv-1(\bmod (p)) \text { is solvable } \Leftrightarrow \operatorname{ord}_{(p)} \theta \text { is even . }
$$

Now

$$
\theta^{\frac{p+1}{2}}=(-1)^{\frac{p+1}{2}} \varepsilon^{p+1} \equiv a(\bmod p)
$$

for some $a \varepsilon Z$ because $G F(p)^{*}=\left\{\psi^{p+1}: \psi \varepsilon G F\left(p^{2}\right)^{*}\right\}$. Applying the nontrival automorphism of $Q(\sqrt{5} \overline{)}$ gives

$$
\bar{\theta}^{\frac{p+1}{2}} \equiv a(\bmod p)
$$

hence

$$
1=(\theta \bar{\theta})^{\frac{p+1}{2}} \equiv a^{2}(\bmod (p)) .
$$

Thus

$$
\theta^{p+1} \equiv a^{2} \equiv 1(\bmod (p))
$$

Consequently $\operatorname{ord}_{(p)} \theta \mid p+1$. Now when $p \equiv-1+2^{j}\left(\bmod 2^{j+1}\right)$ we have

$$
\theta^{\frac{p+1}{2^{\prime}}} \equiv 1(\bmod (p)) \Leftrightarrow \operatorname{ord}_{(p)} \theta \text { is odd }
$$

We now decompose 


$$
S_{B}=\bigcup_{j=1}^{\infty} S_{B}^{(j)}
$$

where

$$
S_{B}^{(1)}=\left\{p: p \equiv 1(\bmod 4) \text { and } p \in S_{B}\right\} \text {. }
$$

and

$$
S_{B}^{(j)}=\left\{p: p \equiv-1+2^{j}\left(\bmod 2^{j+1}\right) \text { and } p \varepsilon S_{B}\right\} \text {. }
$$

We complete case 2 with the following lemma.

Lemma 3.2. (1) $S_{B}^{(1)}$ is empty.

(2) For $j \geq 2$ all $S_{B}^{(j)}=\left\{p: p \equiv-1+2^{j}\left(\bmod 2^{j+1}\right)\right.$ and $\left.p \equiv \pm 2(\bmod 5)\right\}$ and $S_{B}^{(j)}$ has density $2^{-j}$.

Proof. (1) When $j=1$ we have

$$
\theta^{\frac{p+1}{2}} \equiv 1(\bmod (p)) \Leftrightarrow \operatorname{ord}_{(p)} \theta \text { is odd }
$$

Now $\theta=-\varepsilon^{2}$ so

$$
\theta^{\frac{p+1}{2}} \equiv\left(-\varepsilon^{2}\right)^{\frac{p+1}{2}} \equiv-\varepsilon^{p+1}(\bmod (p))
$$

We claim

$$
\varepsilon^{p+1} \equiv-1(\bmod (p))
$$

which with (3.11) shows $\theta^{\frac{p+1}{2}} \equiv 1(\bmod (p))$ and so by (3.10) $\operatorname{ord}_{p} \theta$ is odd and $S_{B}^{(1)}$ is empty.

To prove the claim, set

$$
\varepsilon^{\frac{p+1}{2}} \equiv \phi(\bmod (p))
$$

so

$$
\varepsilon^{p+1} \equiv \phi^{2}(\bmod (p))
$$

By conjugation $\bar{\varepsilon}^{\frac{p+1}{2}} \equiv \bar{\phi}(\bmod p)$ and $\varepsilon \bar{\varepsilon}=-1$ so that 


$$
-1=(-1)^{\frac{p+1}{2}} \equiv(\varepsilon \bar{\varepsilon})^{\frac{p+1}{2}} \equiv \phi \bar{\phi}(\bmod (p)) .
$$

By $(3.8) \varepsilon^{p+1} \equiv \pm 1(\bmod (p))$. We suppose $\varepsilon^{p+1} \equiv 1(\bmod (p))$ and get a contradiction. In that case (3.12) gives $\phi^{2} \equiv 1(\bmod (p))$, hence $\phi \equiv \pm 1(\bmod (p))$. Hence $\phi \equiv \bar{\phi}(\bmod (p))$ and (3.13) now gives

$$
\phi^{2} \equiv-1(\bmod (p))
$$

the desired contradiction.

(2) We must show that in the case $j \geq 2$ for any $p \equiv-1+2^{j}\left(\bmod 2^{j+1}\right)$ and $p \equiv \pm 2(\bmod 5)$ we claim $\operatorname{ord}_{(p)} \theta$ is even. We argue by contradiction. Suppose $\operatorname{ord}_{(p)} \theta$ were odd, so that by (3.8) we have

$$
\theta^{\frac{p+1}{2^{j}}} \equiv 1(\bmod (p))
$$

Set

$$
\varepsilon^{\frac{p+1}{2^{\prime}}} \equiv \phi(\bmod (p))
$$

and observe (3.14) gives

$$
-\phi^{2} \equiv 1(\bmod (p))
$$

Now

$$
\bar{\varepsilon}^{\frac{p+1}{2^{j}}} \equiv \bar{\phi}(\bmod (p))
$$

and

$$
-1=(-1)^{\frac{p+1}{2^{j}}} \equiv(\varepsilon \bar{\varepsilon})^{\frac{p+1}{2^{j}}} \equiv \phi \bar{\phi}(\bmod (p)) .
$$

Now by $(3.15) \quad \phi^{2} \equiv-1(\bmod (p)) \quad$ and $\quad$ since $p \equiv 3(\bmod 4) \quad \bar{\phi} \equiv-\phi(\bmod (p))$. Hence $\phi \bar{\phi} \equiv-\phi^{2} \equiv 1(\bmod (p))$, contradicting $(3.16)$.

As in the proof of Theorem A Lemma 3.2 implies the density of primes in $S_{B}$ is $\sum_{j=2}^{\infty} 2^{-j-1}=\frac{1}{4}$. This proves Theorem B. 


\section{Proof of Theorem C (Sketch)}

We have

$$
V_{n}=\left(\frac{1}{2}+\frac{1}{6} \sqrt{ }-\overline{3}\right)\left(\frac{5}{2}+\frac{1}{2} \sqrt{ }-\overline{3)^{n}}+\left(\frac{1}{2}-\frac{1}{6} \sqrt{ }-\overline{-3}\right)\left(\frac{5}{2}-\frac{1}{2} \sqrt{ }-\overline{3}\right)^{n} .\right.
$$

Letting $\alpha=\frac{1}{2}+\frac{1}{6} \sqrt{ }-3$ and $\gamma=\frac{5}{2}+\frac{1}{2} \sqrt{ }-\overline{3}$ we have

$$
V_{n} \equiv 0(\bmod (p)) \Leftrightarrow \phi^{n} \equiv-\frac{\bar{\alpha}}{\alpha}(\bmod (p))
$$

where $\phi=\frac{\gamma}{\bar{\gamma}}=\frac{11+5 \sqrt{-3}}{14}$ and $-\frac{\bar{\alpha}}{\alpha}=\frac{-1+\sqrt{-3}}{2}$ is a cube root of unity. Hence (4.1) gives

$$
p \text { divides } V_{n} \text { for some } n \geq 0 \Leftrightarrow \operatorname{ord}_{(p)} \phi \equiv 0(\bmod 3) \text {. }
$$

We consider separately the cases in which $(p)$ splits completely or remains inert in $Q(\sqrt{ }-\overline{3})$.

Case 1. $p \equiv 1(\bmod 3)$.

Then $(p)=\pi \bar{\pi}$ in $Z\left[\frac{1+\sqrt{-3}}{2}\right]$. Now as in Theorem B we have

$$
\operatorname{ord}_{(p)} \phi \equiv 0(\bmod 3) \Leftrightarrow \operatorname{ord}_{\pi} \phi \equiv 0(\bmod 3)
$$

using the fact that $\phi \bar{\phi}=1$. Now let $3^{j} \| p-1$, and observe that in this case

$$
\operatorname{ord}_{\pi} \phi \neq 0(\bmod 3) \Leftrightarrow \phi^{\frac{p-1}{3^{\prime}}} \equiv 1(\bmod \pi)
$$

Then

$$
\begin{aligned}
\theta^{\frac{p-1}{3^{j}}} \equiv 1(\bmod \bar{\pi}) & \Leftrightarrow \pi \text { splits completely in } F_{j}=Q\left({ }^{3^{j}} \sqrt{1}-{ }^{3^{j}} \sqrt{\theta} \overline{)} / Q\left({ }^{3} \sqrt{1} \overline{)}\right.\right. \\
& \Leftrightarrow(p) \text { splits completely in } F_{j} / Q .
\end{aligned}
$$

Hence the density of primes satisfying (4.6) is $\left[F_{j}: Q\right]^{-1}=\left(2 \cdot 3^{2 j-1}\right)^{-1}$, and the density $d_{j}$ of primes with $3^{j} \| p-1$ and (4.4) holding is

$$
d_{j}=2\left(2 \cdot 3^{j}\right)^{-1}-\left(2 \cdot 3^{2 j-1}\right)^{-1}
$$


The total contribution of such primes has density

$$
D_{1}=\sum_{j=1}^{\infty} d_{j}=\frac{5}{16}
$$

Case 2. $p \equiv 2(\bmod 3)$.

Then $(p)$ is inert in $Z\left[\frac{1+\sqrt{-3}}{2}\right]$ and, as in Theorem B we have

$$
\phi^{p+1} \equiv 1(\bmod (p))
$$

and if $3^{j} \| p+1$ then

$$
\operatorname{ord}_{(p)} \phi \neq 0(\bmod 3) \Leftrightarrow \phi^{\frac{p+1}{3^{\prime}}} \equiv 1(\bmod (p))
$$

Now we have

$$
\phi^{\frac{p+1}{3^{j}}} \equiv 1(\bmod (p)) \Leftrightarrow p \equiv 2(\bmod 3) \text { and }(p) \text { splits completely in } F_{j} / Q(\sqrt{ }-\overline{3})
$$

We claim that the set of primes defined by the right side of (4.9) has density $\left(2 \cdot 3^{2 j-1}\right)^{-1}$. To verify this, one checks that $F_{j} / Q$ is Galois over $Q$ with Galois group of order $2 \cdot 3^{2 j-1}$, that the splitting condition (4.8) on primes in $F_{j} / Q$ corresponds exactly to the Artin symbol $\left[\frac{F_{j} / Q}{(p)}\right]$ being the conjugacy class $<\sigma>$, where $\sigma$ is the unique element of order two in $\mathrm{Gal}\left(F_{j} / Q\right)$. Then the Chebotarev density theorem implies that the set of primes in (4.8) has density $\left[F_{j}: Q\right]^{-1}=\left(2 \cdot 3^{2 j-1}\right)^{-1}$, as claimed.

Hence the density $d_{j}^{*}$ of primes with $3^{j} \| p+1$ and (4.4) holding is

$$
d_{j}^{*}=2\left(2 \cdot 3^{j}\right)^{-1}-\left(2 \cdot 3^{2 j-1}\right)^{-1}
$$

and the total density of such primes is

$$
D_{2}=\sum_{j=1}^{\infty} d_{j}^{*}=\frac{5}{16}
$$

\section{Acknowledgement}

I am indebted to J. P. Serre for informing me of the work of Hasse. 


\section{References}

[1] A. Aigner, Bemerkung und Lösung zum Problem 29, Elem d. Math. 15 (1960), 66-67.

[2] B. J. Birch, Cyclotomic Fields and Kummer Extensions, in: Algebraic Number Fields (J. W. S. Cassels and A. Fröhlich, Eds.), Academic Press, London 1967, 85-93.

[3] H. Hasse, Uber die Dichte der Primzahlen p, fur die vorgegebene ganzrationale Zahl $a \neq 0$ von durch eine vorgegebene Primzahl $\gg \neq 2$ teilbarer bzw. unteilbarer Ordnung mod p ist., Math. Ann. 162 (1965), 74-76.

[4] H. Hasse, Uber die Dichte der Primzahlen p, für die eine vorgegebene ganzrationale Zahl $a \neq 0$ von gerader bzw. ungerader Ordnung mod p ist., Math. Annalen 166 (1966) 19-23.

[5] J. C. Lagarias, Sets of primes determined by systems of polynomial congruences, Illinois J. Math. 27 (1983), 224-235.

[6] S. Lang, Algebraic Number Theory, Addison-Wesley Publ. Co., New York 1970.

[7] R. R. Laxton, On groups of linear recurrences I, Duke Math J. 26 (1969) 721-736.

[8] R. R. Laxton, On groups of linear recurrences II. Elements of Finite Order, Pacific J. Math. 32 (1970) 173-179.

[9] R. R. Laxton, Arithmetic Properties of Linear Recurrences, in: Computers and Number Theory (A. O. L. Atkin and B. J. Birch, Eds.), 119-124.

[10] W. Narkiewicz, Elementary and Analytic Theory of Algebraic Numbers, Polish Scientific Publishers, Warsaw 1974.

[11] R. W. K. Odoni, A Conjecture of Krishnamurthy on Decimal Periods and Some Allied Problems, J. Number Theory 13 (1981) 303-319.

[12] W. Sierpinski, Sur une decomposition des numbers premiers en deux classes, Collect. Math. 10 (1958), 81-83. (Also: Problem 29, Elem. d. Math. 14 (1959), 60.) 
[13] P. J. Stephens, Prime divisors of second order linear recurrences I., J. Number Theory 8 (1976), 313-332.

[14] P. J. Stephens, Prime divisors of second order linear recurrences II, J. Number Theory 8 (1976), $333-345$.

[15] M. Ward, Prime divisors of second order recurring sequences, Duke Math. J. 21 (1954), 178-188.

[16] M. Ward, The prime divisors of Fibonacci numbers, Pacific J. Math. 11 (1961), 379-386. 


\section{THE SET OF PRIMES DIVIDING THE LUCAS NUMBERS HAS DENSITY 2/3}

\section{J. C. Lagarias}

Bell Laboratories

Murray Hill, NJ 07974

ABSTRACT

Dedicated to the memory of Ernst Straus

The Lucas numbers $L_{n}$ are defined by $L_{0}=2, L_{1}=1$ and the recurrence $L_{n}=L_{n-1}+L_{n-2}$. The set of primes $S_{L}=\left\{p\right.$ : $p$ divides $L_{n}$ for some $\left.n\right\}$ has density $2 / 3$. Similar density results are proved for sets of primes $S_{U}=\left\{p: p\right.$ divides $U_{n}$ for some $\left.n\right\}$ for certain other special second-order linear recurrences $\left\{U_{n}\right\}$. The proofs use a method of Hasse. 